\title{
A two-stage planning and control model toward Economically Adapted Power Distribution Systems using analytical hierarchy processes and fuzzy optimization
}

\author{
Gustavo Schweickardt ${ }^{\mathrm{a}, *}$, Vladimiro Miranda ${ }^{\mathrm{b}, 1}$ \\ a Instituto de Economía Energética, Fundación Bariloche, Centro Atómico Bariloche - Pabellón 7, Av. Bustillo km 9500,8400 Bariloche, Argentina \\ ${ }^{\mathrm{b}}$ INESC Porto, Instituto de Engenharia de Sistemas e Computadores do Porto and FEUP, Faculdade de Engenharia da Universidade do Porto, \\ R. Dr. Roberto Frias, 378, 4200-465 Porto, Portugal
}

\section{A R T I C L E I N F O}

\section{Article history:}

Received 16 February 2007

Received in revised form 12 February 2009

Accepted 13 March 2009

Available online $\mathrm{xxxx}$

\section{Keywords:}

Fuzzy Dynamic Programming

Analytic Hierarchy Processes

Dynamic Adaptation Vector

Economically Adapted Distribution System

\begin{abstract}
A B S T R A C T
This work presents a model to evaluate the Distribution System Dynamic De-adaptation respecting its planning for a given period of Tariff Control. The starting point for modeling is brought about by the results from a multi-criteria method based on Fuzzy Dynamic Programming and on Analytic Hierarchy Processes applied in a mid/short-term horizon (stage 1). Then, the decision-making activities using the Hierarchy Analytical Processes will allow defining, for a Control of System De-adaptation (stage 2), a Vector to evaluate the System Dynamic Adaptation. It is directly associated to an eventual series of inbalances that take place during its evolution.
\end{abstract}

(c) 2009 Elsevier Ltd. All rights reserved.

\section{Introduction}

The definition of an Economically Adapted Power Distribution System (EAPDS) (sometimes referred as Adapted Distribution Network) is a concept that Regulatory Authority, after the de-regulation of electricity industry, has coined and applied without both theoretical and methodological bases. Following the Neoclassical Economics Paradigm, only considering system expansion and operation costs, require that the total cost in some system horizon planning, becomes minimum. Any departure from this condition, once the planning is available, is judged as a de-adaptation. Therefore, all possible system de-adaptation will be static, ignoring the historical-evolutive nature of the system. This lack in the concept and in their operational definition, requires, of course, the previous knowledge of system planning. However, this planning is not enough to obtain a definition operationally useful, as measure to an economically de-adapted system. Fourth reasons, at least, can be observed for it: (a) the planning facing a multi-criteria optimization problem and several of such criteria, have not economic assessment (environmental cost, for example); (b) many or all control variables involved in the planning, have uncertainties, the nat-

\footnotetext{
* Corresponding author. Tel./fax: +54 2944462500.

E-mail addresses: gustavoschweickardt@ciudad.com.ar (G. Schweickardt), vmiranda@inescporto.pt (V. Miranda).

1 Tel.: +35122 2094230; fax: +351222094150
}

ure of which is not stochastic and, then, its treatment for any classical optimization model is very difficult; (c) suppose that all criteria have an economic opportunity cost associated, and the system control variables are deterministic: still, the departure for evaluating the system de-adaptation will be static, and, in practice, evaluated at the beginning of tariff control period; (d) a uniform criteria to departure definition and its implementation do not exist (could be and over-cost in equipment, because the demand was not the forecasted/predicted, or another arbitrary criteria). Under conditions (c) and (d), the concept is applied, commonly, for Regulatory Authority in countries such as Argentina, Chile, Spain and Portugal.

This paper presents a formal solution model, in the framework to Fuzzy Dynamic Programming (FDP) and Analytic Hierarchy Processes (AHP) techniques, in two stages: the first stage, corresponding to the Distribution System Planning in the mid/short-term, considering available the long-term planning. The second stage, corresponding to the Control of System De-adaptation.

Planning FDP technique is based on the principles optimally proposed by [1] and [2].

Control of the System De-adaptation, required the introduction of the technique AHP $[3,4]$ which completes the model by establishing a Priority Vector that is associated to the criteria (seen as system attributes) that define the aptitude for each alternative of solution. This combined model is intended for: (a) developing the theoretical aspects required to define and, operationally, introduce 


\section{Nomenclature}

\section{Acronyms}

AHP Analytic Hierarchy Processes

EAPDS Economically Adapted Power Distribution System

ESNQC Energy Supplied in Non-Quality Conditions, vinculated to Out-of-Supply Voltage attribute

FDP Fuzzy Dynamic Programming

FI frequency of interruption, vinculated to Interruption Rate attribute

GC Global Cost of Distribution System

GPL Global Power Losses of Distribution System

NSE Non-Supplied Energy

OSV Out-of-Supply Voltage attribute

PDS Power Distribution Planning

\section{Symbols}

$A i \quad i$ th variable-attribute of system

$\{A i\} \quad$ fuzzy set associated to $i$ th variable-attribute

$a_{i \text { ref }} \quad$ value of reference to $A i$

$a_{i} \quad$ value-instance of $A i$

$a_{i j} \quad$ generic element of Judgment Matrix to the set of atttributes $\mathbf{A}$

CCA Random Consistency Coefficient

$E^{k} \quad$ state vector corresponding to system in the stage $k$ of planning

$e_{i}^{k} \quad i$ th state/component of $E^{k}$
$I C_{\text {Saaty }}$ Saaty's Consistency Index

$I_{D} \quad$ De-Adaptation Index

$\boldsymbol{M P}_{A} \quad$ Judgment Matrix to the set of attributes $\mathbf{A}$

$p_{i} \quad i$ th component of $\boldsymbol{V}^{\text {Yager }}$ corresponding to ith fuzzy set $\{A i\}$

$R C \quad$ Consistency Ratio related to $I C_{\text {Saaty }}$ and CCA

$R_{D} \quad$ Adaptation Ratio

$\left[T^{*}\right] \quad$ resulting trajectory from FDP

$u_{i} \quad$ departure variable of $A i$

$V_{\text {Adap }} \quad$ Dynamic Adaptation Vector for trajectory [ $\left.T^{*}\right]$

$\boldsymbol{V}_{P} \quad$ Perron's eigenvector of Judgment Matrix. Priority Vector in the context of AHP

$\boldsymbol{V}^{\text {Yager }}$ Vector of Exponential Weighers (from Yager) applied on fuzzy set $\{A i\}$

$\lambda_{P} \quad$ Perron's eigenvalue. Maximum and Positive (real) eigenvalue of $\boldsymbol{M} \boldsymbol{P}_{A}$

$\{D\} \quad$ decision fuzzy set defined as the intersection of all $\{A i\}$

$\mu_{\{D\}} \quad$ membership function of $\{D\}$

$\Delta_{\mu_{i D\}}} \quad$ Acceptance Variation of Trajectory from present stage to horizon of planning

$\mu_{\{A i\}}\left(u_{i}\right)$ membership function of $\{A i\}$

$\mu_{\{D\}}\left[T^{*}\right]$ maximizing decision on fuzzy set $\{D\}$, corresponding to resulting trajectory $\left[T^{*}\right]$, referred as Intrinsic Risk of trajectory $\left[T^{*}\right]$

$\Theta_{\text {Ext }} \quad$ Extrinsic Robustness into the problem of decision-making, the intrinsic risk associated to a certain satisfactory solution; and (b) giving a formal treatment to the concept of Adaptation (De-adaptation) of the system.

Therefore, the proposal model will be referred as (FDP + AHP) planning.

For discussion of some results, a Study Case is presented. The system under analysis is the power distribution system for the city of San Carlos de Bariloche, Province of Rio Negro, Argentina, for which a 10-year horizon has been considered, starting in 1998, for the long-term. The mid/short-term coincides with the 5-year tariff period 1998-2002.

\section{Power distribution systems planning}

As regards the objectives of the present work, such a planning is set forth through a two-stage strategy: the long-term (1-15 years) planning, that is focused on the spatial optimization of the PDS and, the mid/short-term (5-7 years) planning model that emphasizes the time optimization of the PDS.

For the long-term planning, the system optimization has two aims: (a) to minimize the costs associated to building substations and network lines, and their operative costs (Global Costs of the PDS) as well; to minimize the non-quality (that is, the absence of good quality) of electrical energy, pondered through the Non-Supplied Energy (NSE) under contingency.

The two main constraints are: (a) radiality, and (b) coherence in equipment selection and combinable variants, which ensure the coherence among the equipment of the PDS, by defining states between consecutive stages. Other three constraints, regarded implicitly included, are: (a) restriction on demand supply; (b) restriction on the limits of power supply capacity of substations, and on feeders capacity; and (c) restriction of maximum permissible voltage drops. With these considerations, the goal of the strategy is to achieve a set of solution trajectories admitted as satisfactory, on which the system will be capable to evolve. Restriction (b) allows flexing the choice (combinability) of variants to conform various trajectories on a time-horizon where the uncertainty has decreased. Then, in the short/mid-term planning, multiple objectives/restrictions will take place, whose merits will be evaluated in order to define the best trajectories for the mid or short-term.

\section{Long-term planning: A suggested approach from evolutionary programming}

This work supposes that the long-term planning is available. However, under a hierarchy planning basic principle, the best short/mid-term planning must be driven for the best long-term planning. Therefore, suggestions about the model used for the long-term planning must be made. Classical approaches are frequently used, such as those presented in [5] for substations, and in [6] for conductors sizing. This work suggests a multi-objective model for optimal system expansion in the long-term, by following a pseudo-dynamic multi-stage, and facing the uncertainties of its environments. Specifically, it must focus both on the power lines (conductors sizing) on the system substations. The suggested technique, is an approach from evolutionary programming, in particular, using Genetic Algorithms, as coherent alternative facing the type of uncertainties (non-stochastic) inherent to system. See Refs. [7] and [8].

\section{Stage 1. Mid/short-term planning: an application of fuzzy dynamic programming}

\subsection{Main elements of the model}

With the FDP technique, the fuzzy sets, associated to the $n$-attributes with which the aptitude of the system is defined, are mapped on the same fuzzy space of decision-making. To this aim, such sets are built upon the departures from a given reference value of the evaluated magnitude. 
For a given attribute $A i$, whose value for a given state (instance) is $a_{i}$, the departure with respect to certain reference value $a_{i r e f}$, from which the associated fuzzy set is constructed, will be:

$u_{i}=\frac{\left|a_{i}-a_{i r e f}\right|}{a_{i r e f}}$

and the corresponding fuzzy set, $\{A i\}$, whose membership function is $\mu_{\{A i\}}\left(u_{i}\right)$, is defined as:

$\{A i\}=\sum_{i=1}^{n} \mu_{\{A i\}}\left(u_{i}\right) / u_{i} ; \quad u \in U$

following the notation for fuzzy sets [2], where each variable $u$ belongs to discurse universe $U$.

Once the fuzzy sets associated to the departures have been defined, the next step is to introduce the AHP technique for which the judgment matrix $\boldsymbol{M} \boldsymbol{P}_{A}$ is conformed. If $\mathbf{A}$ is the set of attributes of system, then the $\boldsymbol{M} \boldsymbol{P}_{A}$ elements, $a_{i j}$, result from a pairwise comparison of preferences for all attributes in the set $\mathbf{A}$. By following the dominance scale proposed by [3], where $a_{i j}$ is an integer in the interval $[1,10]\left(a_{j i}\right.$ is a rational number equal to $\left.1 / a_{i j}\right), \boldsymbol{M} \boldsymbol{P}_{A}$ is defined as:

$$
\boldsymbol{M P}_{A}=\left(\begin{array}{llll}
1 & a_{12} & \cdots & a_{1 n} \\
a_{21} & 1 & \cdots & a_{2 n} \\
\vdots & \vdots & \ldots & \ldots \\
a_{n 1} & a_{n 2} & \vdots & 1
\end{array}\right) \text { with }\left\{\begin{array}{l}
a_{i j}>0 \\
a_{j i}=\frac{1}{a_{i j}}
\end{array} \quad \forall i, j=\{1 \ldots n\}\right.
$$

Perron's Theorem [9] warrants, for such a matrix, the existence of a dominant, positive eigenvalue $\lambda_{P}$ and its corresponding eigenvector $\boldsymbol{V}_{P}$, also with positive components. Both elements have fundamental importance for this model. The following inequality will be true:

$\lambda_{P} \geqslant n$

and only if matrix $\mathbf{M P}_{A}$ shows consistent judgements, $\lambda_{P}=n$.

The consistency condition [4], is expressed on the elements of $\boldsymbol{M P}_{\mathrm{A}}$ as follows:

$a_{i k}=a_{i j} x a_{j k} ; \quad \forall i, j, k=1 \ldots n$

Condition (5) is introduced in the form of a Saaty's Consistency Index to ponder the transitivity on preferences between the system attributes, that had been established for the planner in $\mathbf{M P}_{A}$ :

$I C_{\text {Saaty }}=\left(\frac{\lambda_{P}-n}{n-1}\right)$

Index $I C_{\text {Saaty }}$ may seem to be strict, if the number of attributes is greater than 5 (five). In this way, the Consistency Condition gets reformulated by using a Random Consistency Coefficient, CCA $>1$, if $n_{A} \geqslant 6[4]$, which can be stated as:

$R C=\frac{I C_{\text {Saaty }}}{C C A} \leqslant 0.1$

RC: Consistency Ratio.

Associated to Perron's eigenvalue, $\lambda_{P}$, Perron's eigenvector, $\boldsymbol{V}_{P}$, satisfies the Hierarchical Composition Principle [4], which is defined as (from the eigenvector (V) - eigenvalue (c) expression, for a squared matrix):

$\boldsymbol{M P} \boldsymbol{P}_{A} \boldsymbol{x}=\boldsymbol{C} \times \boldsymbol{V}$

if $c=\lambda_{P}$, and, consequently, $\boldsymbol{V}=\boldsymbol{V}_{P}$.

$\boldsymbol{V}_{P}$ results to be the Priority Vector on the preferences established between the system attributes. Therefore, if its form is changed to Yager's form [10], by normalizing and multiplying it by the order of the Judgment Matrix, a Vector of Exponential Weighers, $\boldsymbol{V}^{\text {Yager }}$ will be obtained. This vector will be applied to the fuzzy sets associated to the various attributes in order to modify their importance in the fuzzy optimization.

\subsection{Fuzzy dynamic programming and formal model for mid/short- term}

Fig. 1, present one step in the transition between the stage $k$ to $k-1$ in a forward dynamic [1]. The criteria followed for each transition (each state $(j, k-1)$ to state $(1, k))$ are the $\operatorname{Max}\{\operatorname{Min}\{\ldots\}\}$ of the membership function value of each system attribute $(1 \ldots n)$, $\mu_{\{A i\}}\left(u_{i}\right)[2]$. Considering the Vector of Exponential Weighers, $\boldsymbol{V}^{\text {Yager }}$, calculated as explained above, each $\mu_{\{A i\}}\left(u_{i}\right)$ will be pondered as the preferences established in the Judgment Matrix $\boldsymbol{M P}_{A}$. Such exponential weight, expressed as $\left[\mu_{\{A i\}}\left(u_{i}\right)\right]^{p_{i}}$, where $p_{i}$ is the $i$ th component of $\boldsymbol{V}^{\text {Yager }}$, has an effect of contraction or expansion on the corresponding fuzzy set, according to the value of $p_{i}$ : if $p_{i}>1$, then there will be contraction, and the fuzzy set $\left[\mu_{\{A i\}}\left(u_{i}\right)\right]^{p_{i}}$ will be given a higher importance than $\mu_{\{A i\}}\left(u_{i}\right)$ in the $\operatorname{Max}\{\operatorname{Min}\{\ldots\}\}$ optimization criteria. The opposite occurs when $p_{i}<1$, where there will be expansion of fuzzy set.

If the static optimization (between two stages only) presented in Fig. 1 , is extended for each state $\left[\forall e_{j}^{k-1} \in E^{k-1}\right]$ and each state $\left[\forall e_{i}^{k} \in E^{k}\right]$, where $E^{k-1}\left(E^{k}\right)$ is the state vector for stage $k-1(k)$, the corresponding fuzzy optimization (formal model for mid/ short-term planning) is stated as:

$$
\begin{aligned}
& {\left[\forall e_{i}^{k} \in E^{k}\right] ; \quad k=1,2 \ldots N:} \\
& \mu_{\{\boldsymbol{D}\}}^{*}(i, k)=\operatorname{Max}\left\{\begin{array}{ll}
\left.\operatorname{Min}_{\left[\forall e_{j}^{k-1} \in E^{k-1}\right.}\right] & \left\{\begin{array}{l}
{\left[\mu_{\{A 1\}}(j, k-1) ;(i, k)\right]^{p_{1}} ;} \\
{\left[\mu_{\{A 2\}}(j, k-1) ;(i, k)\right]^{p_{2}} ; \cdots ;} \\
{\left[\mu_{\{A n\}}(j, k-1) ;(i, k)\right]^{p_{n}} ; \mu_{\{D\}}^{*}(j, k-1)}
\end{array}\right\}
\end{array}\right\}
\end{aligned}
$$

subject to:

(1) $\boldsymbol{M} \boldsymbol{P}_{A}$ invariant in $\left[T^{*}\right]$ (the preferences between the system attributes, cannot be changed)

(2) $\mu_{\{D\}}\left[T^{*}\right] \geqslant\left[1-\Theta_{E x t}\right]$

where the membership value $\mu_{\{D\}}^{*}(i, k)$ is maximized in the decisionmaking fuzzy set, $\{D\}$, for every state $\left[\forall e_{i}^{k} \in E^{k}\right]$, on the horizon of $\mathbf{N}$ stages; $p_{1}, p_{2}, \ldots, p_{n}$ are the Yager Weighers of vector $\boldsymbol{V}^{\text {Yager }} ;\left[T^{*}\right]$ is the resulting Trajectory and $\mu_{\{D\}}\left[T^{*}\right]=\mu_{\{D\}}^{*}(i=1, k=n)$ in the unique state $i$, for the last stage $k=n$, is the value obtained from the recursive application of (9). $\mu_{\{D\}}$ will be referred as Intrinsic Risk of [ $\left.T^{*}\right]$. Constraint (1) on invariability in $\boldsymbol{M P}_{A}$ imposes that the Yag-

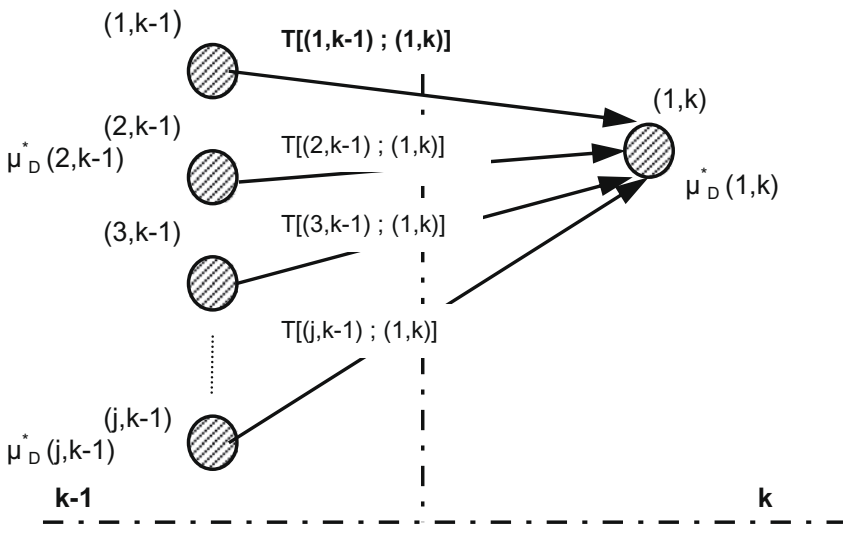

Fig. 1. Transition between stage $k-1$ to stage $k$ in the FDP forward. 
er Weighers remain unaltered on the resulting Trajectory [ $\left.T^{*}\right]$. Constraint (2) will be called the Extrinsic Risk Constraint [11,12]. Is externally fixed by planner by mean a value, $\Theta_{\text {Ext }}$, between $[0,1]$, as a complement to the less-acceptable membership of fuzzy set $\{D\}$. The parameter $\Theta_{E x t}$ is called Extrinsic Robustness. A high level of robustness implies a low level of risk, but a high level of uncertainties [13].

The link:

$\mu_{\{D\}}^{*}(j, k-1) \rightarrow \mu_{\{D\}}^{*}(i, k)$

constitutes the dynamic condition of the fuzzy optimization process.

\section{Stage 2. Control of system de-adaptation: the Dynamic Adaptation Vector}

There are some theoretical concepts which required explanation, before its introduction in the second stage of proposal model and are developed in the following paragraphs.

\subsection{The De-Adaptation Index: First component of Dynamic Adaptation} Vector

The Dynamic Adaptation Vector is proposed in the second stage of model, in order to evaluate the de-adaptation of the real system (or present state system) respecting the projected system. It has two components: the first one is related to the variation that the system may present as regards its aptitude attributes, as a consequence of change in the preferences among such attributes. This work, is called the De-Adaptation Index, and is defined as:

$I_{D}^{[k, P r e]}=\frac{\left(\frac{\lambda_{P}^{[k, P r e]}-n}{n-1}\right)}{C C A}$

where $k$ is the present stage of evaluation; $\lambda_{p}^{[k, P r e]}$ is Perron's eigenvalue for the present judgments matrix, $M P_{A}^{[k, P r e]}$. Its construction is proposed as follows, satisfying symmetrical condition in (3):

$M P_{A}^{[k, \text { Pre }]}=\left(\begin{array}{cccc}{[1]} & \ldots & \ldots & {\left[\frac{p_{n}^{[\text {Proj }]}}{p_{1}^{k, P r e]}}\right]} \\ \vdots & \vdots & \ldots & \vdots \\ \vdots & \vdots & \ddots & \vdots \\ {\left[\frac{p_{1}^{[\text {Proj }]}}{p_{n}^{k, \text { Pre }}}\right]} & \ldots & \ldots & {[1]}\end{array}\right)$

where Pre refers to the present stage $[k$, Pre] and Proj the projected stage [Proj]. Depending on the stage of evaluation, $k$, the present $V^{\text {Yager }}$ will be changed, as well as $\boldsymbol{M} \boldsymbol{P}_{A}$ (expression (12)); then:

$$
V^{\text {Yager }[k, \text { Pre }]}=\left(\begin{array}{c}
p_{1}^{[\text {Proj }]} \times \frac{\log \left\{\mu_{A 1[k, P r o j}\right\}}{\log \left\{\mu_{A 1[k, P r e}\right\}} \\
p_{2}^{[\text {Proj }]} \times \frac{\log \left\{\mu_{A 2[k, P r o j}\right\}}{\log \left\{\mu_{A 2[k, P r e}\right\}} \\
\vdots \\
p_{n}^{[P r o j]} \times \frac{\log \left\{\mu_{A n[k, P r o j}\right\}}{\log \left\{\mu_{A n, k, P r e}\right\}}
\end{array}\right)
$$

results of the present Weighers Vector (in stage $k$ ), obtained from the Principle of Invariance on the Weighed Aptitude, are formulated as follows (notice that projected $\mu_{A i}$, depends on the stage $k$ ):

$\left[\mu_{A i[k, P r e]}\right]^{\left[p_{i}^{[k, P r e]}\right]}=\left[\mu_{A i[k, P r o j]}\right]^{\left[p_{i}^{[\text {Proj }]}\right]} ; \forall i[1 \ldots n] \in A$

which will have to satisfy the present system as regards the projected one. If $\mu_{A i[k \text { Pre }}$ result 1 , then any $p_{i}^{[P r o j]}$ can satisfy (14). In this case, the product $\left[p_{i}^{[P r o j]} \times \mu_{A i k, P r o j]}\right]$ it is adopted as $p_{i}^{[k, P r e]}$. Other particular case is present when $\mu_{A i[k, P r o j]}=1$, then $p_{i}^{[k, P r e]}=p_{i}^{[P r o j]}$.
In (14), each system attribute value for the present stage should be surveyed, and their membership in the associated fuzzy sets should be attained.

The proposal construction in Eq. (12), is based on the following Consistent Judgment Matrix property: if $p_{1}, p_{2}, \ldots, p_{n}$ are the Yager Weighers of vector $\boldsymbol{V}^{\text {Yager }}$, defined externally, then the matrix:

$\boldsymbol{M P}=\left(\begin{array}{cccc}1 & \frac{p_{2}}{p_{1}} & \ldots & \frac{p_{n}}{p_{1}} \\ \frac{p_{1}}{p_{2}} & 1 & \ldots & \frac{p_{n}}{p_{2}} \\ \vdots & \vdots & \ldots & \vdots \\ \frac{p_{1}}{p_{n}} & \frac{p_{2}}{p_{n}} & \cdots & 1\end{array}\right)$

is a consistent matrix, and associated to Perron's eigenvalue, $\lambda_{P}=n$.

Such property can be easily demonstrated, applying the consistency condition (5).

As a result from randomness, from (12), a judgements matrix could be attained which is more consistent than the one established in the planning. In this case the resulting Yager's Vector will be the one that will prove the coherence of the process. In general the following setting cases will take place:

(a) $\boldsymbol{M P}_{A}^{[k, P r e]}$ turns to be consistent, but the vector $V^{\text {Yager }[k, P r e]}$ prioritizes the attributes rather inconsistently to the eyes of the planner/regulator. The case will then be one of a consistent deadaptation: $\left(\lambda_{\mathrm{p}}^{[k, \text { Pre }]} \approx n ; V^{\text {Yager }}[k\right.$, Pre $] \neq V^{\text {Yager }}[$ Proj $\left.]\right)$; (b) The situation $\left(\lambda_{P}^{[k, P r e]} \approx n ; V^{\text {Yager }}[k\right.$, Pre $] \neq V^{\text {Yager }}[$ Proj $\left.]\right)$ with consistent $V^{\text {Yager }[k, \text { Pre }]}$ constitutes a consistent adaptation of the system. It will be a perfect one if $\left(\lambda_{P}^{[k, P r e]} \approx n ; V^{\text {Yager }}[k\right.$, Pre $]=V^{\text {Yager }}[$ Proj $\left.]\right)$; (c) Finally, the case $\left(\lambda_{P}^{[k, P r e]} \gg n ; V^{\text {Yager }}[k\right.$, Pre $] \neq V^{\text {Yager }}[$ Proj $\left.]\right)$ with non-consistent $V^{\text {Yag- }}$ ${ }^{e r}[k$,Pre] constitutes a non-consistent de-adaptation respecting the projected system and the rationality of its agents.

The adaptation condition relative to a change of judgments or Adaptation Ratio in the present stage $k$, with respect to the projected stage, proj, will be expressed as:

$$
\begin{aligned}
R_{D}^{[k, P r e]} & =\operatorname{Max}\left\{0 ;\left[I_{D}^{k, P r e]}-R C^{[\text {Proj }]}\right]\right\} \Rightarrow R_{D}^{[k, P r e]} \\
& =0 \rightarrow \text { Perfect adaptation }
\end{aligned}
$$

\subsection{Acceptance variation: Second component of Dynamic Adaptation Vector}

The second component of Dynamic Adaptation Vector, referred as the Acceptance Variation of the present stage, is related to the possibility that the system continues evolving from such a state according to a trajectory with a risk smaller or equal to the extrinsic one. That is, the system should meet constraint 2) of model (9).

The condition is formulated as:

$\Delta_{\mu_{\{D\}}}^{[k, P r e]}=\operatorname{Max}\left\{0 ; \frac{\left\{\Theta_{\{D\}}^{*[k, P r e]}-\Theta_{E x t}\right\}}{\Theta_{E x t}}\right\}$

where

$\Theta_{\{D\}}^{*[k, P r e]}=\left[1-\mu_{\{D\}}^{*[k, P r e]}\right]$

To this, the application of (17) should be re-stated by employing $M P_{A}^{[k, P r e]}$ into Condition (1) of model (9), thus setting forth a new optimization for the period under consideration.

The Adaptation Condition for the system, respecting the extrinsic risk on the new evolution trajectory, is expressed as:

$\Delta_{\mu_{\{D\}}}^{[k, P r e]}=0$

Therefore, the Dynamic Adaptation Vector for a given state $e$ on stage $k$ (present stage) can be expressed as: 
$V_{\text {Adap }}^{[k, \text { Pre }]}=\left[\begin{array}{c}R_{D}^{[k, \text { Pre }]} \\ \Delta_{\mu_{\{D\}}}^{[k, \text { Pre }]}\end{array}\right] \Rightarrow\left[\begin{array}{l}0 \\ 0\end{array}\right] \rightarrow$ Perfect Adaptation Condition

The proposed Adaptation Vector is totally a dynamic parameter because its definition is based on the historical-evolutionary nature of the system. The dynamic adaptation does not need to continue with the same evolution trajectory, neither does it require that the comparing states have the same aptitude, a necessary condition for a static adaptation.

\section{Simulation - A study case}

6.1. Stage 1: planning. System attributes and parameters from judgment matrix and FDP

It is first assumed that the long term of the strategy planning has already been solved through the above-suggested techniques in Section 3. The mid-term planning, in this case, coincident with control tariff period ( 5 years), has been solved through the model (9). In the Appendix the equations and tables of results obtained are presented. Five criteria, seen as attributes in the optimization problem, are considered for the PDS: Global Cost (GC); Non-Supplied Energy (NSE); Interruption Rate (FI); Out-of-Supply Voltage (OSV) and Global Power Losses (GPL). The membership functions are exponential (decreasing), but another form-characteristic may be proposed. One cannot fully describe in the paper all the details of the example, but most relevant steps, following this introduction (helped with Tables), illustrate the results obtained.

Step 1: Definition of Judgment Matrix $\boldsymbol{M} \boldsymbol{P}_{A}$ in the planning (or projected) stage:

\begin{tabular}{|c|c|c|c|c|c|}
\hline$M P_{A}$ & GC & NSE & FI & OSV & GPL \\
\hline GC & 1 & 3 & 3 & 2 & 1 \\
\hline NSE & $1 / 3$ & 1 & 1 & $2 / 3$ & $1 / 3$ \\
\hline FI & $1 / 3$ & 1 & 1 & $2 / 3$ & $1 / 3$ \\
\hline OSV & $1 / 2$ & $3 / 2$ & $3 / 2$ & 1 & $1 / 2$ \\
\hline GPL & 1 & 3 & 3 & 2 & 1 \\
\hline
\end{tabular}

Step 2: The following extrinsic robustness $\Theta_{E x t}=0.18$ has been fixed externally to bind the set (in general) of satisfactory trajectories in the evolution of the system with an extrinsic risk

$$
1-\Theta_{\text {Ext }}=1-0.18=0.82
$$

Step 3: Perron's eigenvalue of $\boldsymbol{M} \boldsymbol{P}_{A}$ and Vector of Yager Weighers: In this simulation, $\boldsymbol{M} \boldsymbol{P}_{A}$ have consistent judments (preferences between attributes) and Perron's eigenvalue result:

$\lambda_{P}^{[P r o j]}=5$

and the Transpose Vector of Yager Weighers:

$V_{T}^{\text {Yager[Proj] }}=\left[\begin{array}{lllll}0.5 & 1.5 & 1.5 & 1 & 0.5\end{array}\right]$

Step 4: Application of Fuzzy Optimization Model (9):

In general there will be an Evolution Trajectory Set $T_{i}^{*}$, that belongs to the set bounded by $\Theta_{E x t}$. It ensures the membership condition (constraint (2) of model (9)):

$$
\mu_{\{D\}}\left[T_{i}^{*}\right] \geqslant\left[1-\Theta_{E x t}\right]
$$

Table 1

Penalties in Urban areas ESNQC [\$/kWh]-Rural [MV], [LV].

$0.05 \leqslant \Delta u^{u}<0.06 \rightarrow 0.013$

$0.06 \leqslant \Delta u^{u}<0.07 \rightarrow 0.026$

$0.07 \leqslant \Delta u^{u}<0.08 \rightarrow 0.039$

$0.08 \leqslant \Delta u^{u}<0.08 \rightarrow 0.052$

$0.09 \leqslant \Delta u^{u}<0.10 \rightarrow 0.070$

$0.10 \leqslant \Delta u^{u}<0.11 \rightarrow 0.086$

$0.11 \leqslant \Delta u^{u}<0.12 \rightarrow 0.100$

$0.12 \leqslant \Delta u^{u}<0.13 \rightarrow 0.300$

$0.13 \leqslant \Delta u^{u}<0.14 \rightarrow 0.700$

$0.14 \leqslant \Delta u^{u}<0.15 \rightarrow 1.100$

$0.15 \leqslant \Delta u^{u}<0.16 \rightarrow 1.400$

$0.16 \leqslant \Delta u^{u}<0.18 \rightarrow 1.800$ $\Delta u^{u} \geqslant 0.18 \rightarrow 2.000$

Table 2

Penalties in Rural areas.

$0.10 \leqslant \Delta u^{u}<0.11 \rightarrow 0.025$

$0.11 \leqslant \Delta u^{u}<0.12 \rightarrow 0.050$

$0.12 \leqslant \Delta u^{u}<0.13 \rightarrow 0.075$

$0.13 \leqslant \Delta u^{u}<0.14 \rightarrow 0.100$

$0.14 \leqslant \Delta u^{u}<0.15 \rightarrow 0.300$

$0.15 \leqslant \Delta u^{u}<0.16 \rightarrow 0.700$

$0.16 \leqslant \Delta u^{u}<0.18 \rightarrow 1.400$

$\Delta u^{u} \geqslant 0.18 \rightarrow 2.000$

For this simulation, it is considering only one trajectory (corresponding to the maximum value of membership in the fuzzy decision set, $\{D\})$. Applying the equations presented in the Appendix, with the parameters in the membership function for each fuzzy set, $\mu_{A i}\left(u_{i}\right): \alpha=0.5 ; \beta=0.2 ; \delta=0.2 ; \gamma=0.2 ; \rho=0.7$, the results shown in Tables $1-8$, are obtained. Then, the Evaluation Trajectory of the system for all $N=6$ stages of the considered period has attained a maximum membership value

$\mu_{\{D\}}\left[T^{*}\right]=0.9181 \geqslant\left[1-\Theta_{E x t}\right]$

thus satisfying constraint (2) in (9), with (25).

\subsection{Stage 2: control. Computing system dynamic de-adaptation}

The period spanning 1998-2002 was considered for the mid/ short-term planning. When surveying the system data for year $2001(k=\mathrm{IV})$, the present stage is established (see Table 7). In such a Trajectory, the Dynamic Adaptation Vector of (20) will be calculated. For this, the membership of the associated fuzzy sets is computed from the magnitudes evaluated for each system attribute in $(k=\mathrm{IV})$.

By applying condition (14) and considering the projected values for each attribute in such a stage, the new Transpose Vector of Yager's Weighers is attained (expression (13)). Then:

Step 1: Vector of Yager Weighers of $\boldsymbol{M P}_{A}^{[k=I V, P r e]}$ :

$$
V_{T}^{\text {Yager }[k=I V, P r e]}=\left[\begin{array}{lllll}
0.1694 & 1.5 & 1.5 & 0.1342 & 0.3077
\end{array}\right]
$$

Step 2: Judgment Matrix for the present state, $\boldsymbol{M P}_{A}^{[k=I V, P r e]}$, and Perron's eigenvalue:From (12), $\boldsymbol{M P}_{A}^{[k=I V, P r e]}$ is obtained. Its Perron's eigenvalue results:

$$
\lambda_{P}^{[k=I V, P r e]}=9.0084
$$

Step 3: Adaptation Ratio: For five attributes (Table 5), there is a Random Consistency Coefficient:

$$
C C A(5)=\mathbf{0 . 9}
$$


Table 3

Transition Values of Attributes in Planning Stages with FDP. $\mathbf{e}_{\mathbf{i}}$ :states in stage K-1; $\mathbf{e}_{\mathbf{j}}$ :states in stage K; GC [k\$]; NSE [MWh]; GPL [KW].Other Attributes are addimentional. Stage I: 1998; Stage II: 1999; Stage III: 2000; Stage IV: 2001; Stage V: 2002; Stage VI: 2003 (begin of year)

\begin{tabular}{|c|c|c|c|c|c|c|c|c|}
\hline K-1 & $\mathbf{e}_{i}$ & $\mathbf{K}$ & $\mathbf{e}_{j}$ & GC & NSE & FI & OSV & GPL \\
\hline I & 1 & II & 1 & 128.01 & 17050.17 & 0.73 & 0.0453 & 185.40 \\
\hline I & 1 & II & 2 & 153.31 & 7930.59 & 1.19 & 0.0477 & 210.45 \\
\hline I & 1 & II & 3 & 166.80 & 6919.35 & 1.02 & 0.0362 & 150.04 \\
\hline I & 1 & II & 4 & 177.72 & 14994.58 & 0.81 & 0.0403 & 178.90 \\
\hline I & 1 & II & 5 & 189.37 & 9144.42 & 0.50 & 0.0393 & 190.65 \\
\hline II & 1 & III & 1 & 107.89 & 9042.98 & 0.89 & 0.0473 & 150.00 \\
\hline II & 1 & III & 2 & 111.46 & 11656.20 & 0.98 & 0.0392 & 178.65 \\
\hline II & 1 & III & 3 & 139.19 & 17224.94 & 1.02 & 0.0389 & 145.66 \\
\hline II & 1 & III & 4 & 230.77 & 10196.59 & 0.89 & 0.0375 & 201.54 \\
\hline II & 2 & III & 1 & 141.37 & 13443.41 & 1.46 & 0.0417 & 186.54 \\
\hline II & 2 & III & 2 & 117.29 & 13117.08 & 0.64 & 0.0403 & 170.65 \\
\hline II & 2 & III & 3 & 150.31 & 9708.09 & 1.65 & 0.0457 & 164.30 \\
\hline II & 2 & III & 4 & 328.77 & 9083.66 & 0.66 & 0.0399 & 180.43 \\
\hline II & 3 & III & 1 & 172.33 & 17377.21 & 1.43 & 0.0405 & 193.45 \\
\hline II & 3 & III & 2 & 124.42 & 10094.92 & 0.94 & 0.0436 & 158.69 \\
\hline II & 3 & III & 3 & 204.09 & 9761.17 & 1.49 & 0.0404 & 193.21 \\
\hline II & 3 & III & 4 & 225.18 & 16849.79 & 1.31 & 0.0373 & 154.68 \\
\hline II & 4 & III & 1 & 133.43 & 10722.77 & 1.01 & 0.0402 & 180.35 \\
\hline II & 4 & III & 2 & 121.05 & 18284.90 & 0.57 & 0.0394 & 175.34 \\
\hline II & 4 & III & 3 & 186.81 & 18279.32 & 1.38 & 0.0410 & 160.04 \\
\hline II & 4 & III & 4 & 302.12 & 11088.98 & 1.60 & 0.0483 & 165.98 \\
\hline II & 5 & III & 1 & 98.95 & 18324.34 & 0.90 & 0.0474 & 190.02 \\
\hline II & 5 & III & 2 & 158.29 & 17852.25 & 0.79 & 0.0416 & 201.54 \\
\hline II & 5 & III & 3 & 190.68 & 14236.06 & 1.31 & 0.0448 & 178.54 \\
\hline II & 5 & III & 4 & 213.78 & 8451.13 & 1.62 & 0.0386 & 164.66 \\
\hline III & 1 & IV & 1 & 75.97 & 7059.08 & 1.24 & 0.0451 & 130.02 \\
\hline III & 1 & IV & 2 & 104.08 & 16356.60 & 1.56 & 0.0471 & 125.43 \\
\hline III & 1 & IV & 3 & 137.75 & 16652.26 & 1.32 & 0.0456 & 150.08 \\
\hline III & 1 & IV & 4 & 170.10 & 7119.08 & 1.04 & 0.0516 & 135.98 \\
\hline III & 2 & IV & 1 & 110.66 & 14618.64 & 1.60 & 0.0506 & 115.64 \\
\hline III & 2 & IV & 2 & 103.46 & 17963.78 & 1.57 & 0.0468 & 105.66 \\
\hline III & 2 & IV & 3 & 130.95 & 8184.89 & 0.97 & 0.0512 & 121.45 \\
\hline III & 2 & IV & 4 & 162.53 & 11248.05 & 0.80 & 0.0481 & 140.08 \\
\hline III & 3 & IV & 1 & 136.32 & 17822.66 & 1.07 & 0.0480 & 109.12 \\
\hline III & 3 & IV & 2 & 107.65 & 8612.47 & 0.87 & 0.0491 & 135.08 \\
\hline III & 3 & IV & 3 & 128.15 & 16485.70 & 0.93 & 0.0521 & 127.41 \\
\hline III & 3 & IV & 4 & 129.30 & 15456.34 & 1.48 & 0.0530 & 140.09 \\
\hline III & 4 & IV & 1 & 128.53 & 15233.89 & 1.33 & 0.0491 & 112.10 \\
\hline III & 4 & IV & 2 & 129.27 & 10873.81 & 1.45 & 0.0441 & 101.23 \\
\hline III & 4 & IV & 3 & 103.13 & 16075.44 & 0.67 & 0.0461 & 120.09 \\
\hline III & 4 & IV & 4 & 171.22 & 17967.88 & 1.11 & 0.0471 & 123.80 \\
\hline IV & 1 & V & 1 & 125.69 & 18489.06 & 1.39 & 0.0565 & 165.05 \\
\hline IV & 1 & V & 2 & 213.77 & 17949.82 & 2.14 & 0.0590 & 170.09 \\
\hline IV & 1 & V & 3 & 140.57 & 9343.41 & 0.87 & 0.0548 & 163.23 \\
\hline IV & 2 & V & 1 & 236.58 & 16607.82 & 0.89 & 0.0615 & 155.44 \\
\hline IV & 2 & V & 2 & 154.60 & 8797.17 & 1.62 & 0.0515 & 151.02 \\
\hline IV & 2 & V & 3 & 169.42 & 20180.27 & 1.93 & 0.0556 & 155.43 \\
\hline IV & 3 & V & 1 & 125.61 & 17531.08 & 1.31 & 0.0546 & 180.98 \\
\hline IV & 3 & V & 2 & 165.38 & 18050.65 & 1.94 & 0.0539 & 185.03 \\
\hline IV & 3 & V & 3 & 155.81 & 16699.96 & 1.43 & 0.0535 & 170.41 \\
\hline IV & 4 & V & 1 & 182.86 & 19675.06 & 1.25 & 0.0576 & 165.79 \\
\hline IV & 4 & V & 2 & 193.92 & 17445.31 & 0.94 & 0.0601 & 159.65 \\
\hline IV & 4 & V & 3 & 161.59 & 8552.54 & 1.50 & 0.0577 & 180.10 \\
\hline V & 1 & VI & 1 & 126.79 & 11404.11 & 1.56 & 0.0643 & 289.14 \\
\hline V & 2 & VI & 1 & 191.56 & 8429.04 & 1.70 & 0.0583 & 245.35 \\
\hline V & 3 & VI & 1 & 188.30 & 21474.51 & 2.21 & 0.0610 & 268.57 \\
\hline
\end{tabular}

Table 4

Reference Values of Attributes in Planning Stages with FDP.

\begin{tabular}{llllll}
\hline Stage & NSE Ref [MWh] & FI Ref & $I_{\text {OSV Ref }}$ & Min I ISV Ref Ur & Min I OSV Ref Ru \\
\hline II & 11826 & 0.8 & 0.0381 & 0.0205 & 0.0175 \\
III & 12483 & 1.1 & 0.0362 & 0.0215 & 0.0146 \\
IV & 12921 & 1.1 & 0.0401 & 0.0228 & 0.0173 \\
V & 13578 & 1.5 & 0.0441 & 0.0244 & 0.0197 \\
VI & 14673 & 1.5 & 0.0515 & 0.0261 & 0.0253 \\
\hline
\end{tabular}

Table 5

Random Consistency Coefficient, CCA(n).

\begin{tabular}{lllllllll}
\hline 1 & 2 & 3 & 4 & 5 & 6 & 7 & 8 & 9 \\
\hline 0 & 0 & 0.58 & 0.9 & 0.9 & 1.12 & 1.24 & 1.41 & 1.45 \\
\hline
\end{tabular}

The Consistency Ratio corresponding to the projected system (for the entire horizon under study) becomes:

$R C^{\text {Proj }}=0$, because the system exhibits

perfectly consistent preferences

and, considering (32). the De-Adaptation Index for the present stage $[k=\mathrm{IV}]$, is (from (11)):

$I_{D}^{k=I V, P r e]}=\frac{\left(\frac{9.0084-5}{5-1}\right)}{0.9}=1.1134$

Then, the Adaptation Ratio

$R_{D}^{[k=I V, P r e]}=\operatorname{Max}\{0 ;[1.1134-0]\}=1.1134$

It is not thus complied with the adaptation condition relative to a change in judgment (20), and, from Section 5.1, the system could exhibit (it must be judged by the regulatory authority) a non-consistent de-adaptation.

Step 4: Acceptance Variation: The adopted model (FDP + AHP) is again applied by adopting: (a) the present stage as the initial (only one state) $[k=\mathrm{IV}]$, (b) the present Vector of Yager's Weighers, and (c) a dynamics on a horizon of $(k-N)$ remainder stages governed by the same mid/short-term planning. Hence, the following membership value is attained (see Table 8):

$$
\begin{aligned}
\left.\mu_{\{D\}}^{* k \in=V, P r e}\right] & \left.=0.7758 \rightarrow \Theta_{\{D\}}^{* k \in=V, P r e}\right]=1-0.7758 \\
& =0.2242(\text { from }(18))
\end{aligned}
$$

and the Acceptance Variation, the second component of the Dynamic Adaptation Vector, results to be:

$\Delta_{\mu_{\{D\}}}^{[k=I V, P r e]}=\operatorname{Max}\left\{0 ; \frac{\{0.2242-0.18\}}{0.18}\right\}=0.2455$

Therefore, from (19), the adaptation condition of the system, respecting the extrinsic risk on the new evolution trajectory, is not satisfied.

Finally, in order to judge about the possible de-adaptation of the system, the Dynamic Adaptation Vector can be used:

Table 6

\begin{tabular}{|c|c|c|c|c|c|c|c|c|c|c|c|}
\hline Optimal State & $\mu_{D}\left[T^{*}\right]$ & $\mu_{u_{G C}}^{*}$ & GC $[\mathbf{k} \$]$ & $\mu_{u_{N S E}}^{*}$ & NSE [MWh] & $\mu_{u_{F I}}^{*}$ & FI & $\mu_{u_{o s v}}^{*}$ & OSV & $\mu_{u_{G P L}}^{*}$ & GPL [kW] \\
\hline$[1, \mathrm{I}]$ & 1.000 & 1.0000 & 0.000 & 1.0000 & 0.00000 & 1.0000 & 0.000 & 1.0000 & 0.0000 & 1.0000 & 0.000 \\
\hline$[4, \mathrm{II}]$ & 0.9228 & 1.0000 & 177.720 & 0.9228 & 14994.580 & 0.9963 & 0.810 & 0.9774 & 0.0403 & 1.0000 & 178.90 \\
\hline$[1, \mathrm{III}]$ & 0.9228 & 0.9233 & 311.150 & 1.0000 & 10722.770 & 1.0000 & 1.010 & 0.9996 & 0.0402 & 0.9754 & 359.25 \\
\hline$[4$, IV $]$ & 0.9228 & 0.9850 & 481.250 & 1.0000 & 7119.080 & 1.0000 & 1.040 & 0.9665 & 0.0516 & 0.9470 & 495.230 \\
\hline$[2, \mathrm{~V}]$ & 0.9181 & 0.9634 & 675.170 & 0.9181 & 17445.310 & 1.0000 & 0.940 & 0.9669 & 0.0601 & 0.9403 & 654.880 \\
\hline$[1, \mathrm{VI}]$ & 0.9181 & 0.9313 & 866.730 & 1.0000 & 8429.040 & 0.9608 & 1.700 & 0.9998 & 0.0583 & 1.0000 & 900.230 \\
\hline
\end{tabular}

Optimal Trajectory in Planning Stages with FDP. 
Table 7

Values of Attributes and Membership Function (without Yager's Weighers) in Present State, Stage k=IV.

\begin{tabular}{|c|c|c|c|c|c|c|c|c|c|c|}
\hline Present State at Stage & $\mu_{u_{G C}}^{*}$ & GC $[\mathbf{k} \$]$ & $\mu_{u_{N S E}}^{*}$ & NSE [MWh] & $\mu_{u_{F I}}^{*}$ & FI & $\mu_{u_{O S V}}^{*}$ & OSV & $\mu_{u_{G P L}}^{*}$ & GPL [kW] \\
\hline Ref-Min [IV] & & 467.123 & & 12921.000 & & 1.100 & & 0.0401 & & 459.485 \\
\hline [IV] & 0.9146 & 550.5 & 0.9791 & 14280.550 & 0.9837 & 1.190 & 0.7758 & 0.091 & 0.8378 & 575.65 \\
\hline
\end{tabular}

Table 8

Optimal Trajectory in Re-Planning Stages with FDP.

\begin{tabular}{|c|c|c|c|c|c|c|c|c|c|c|c|}
\hline Optimal State & $\mu_{D}\left[T^{*}\right]$ & $\mu_{u_{\mathrm{GC}}}^{*}$ & GC [k\$] & $\mu_{u_{N S E}}^{*}$ & NSE [MWh] & $\mu_{u_{F I}}^{*}$ & FI & $\mu_{u_{\text {OSV }}}^{*}$ & OSV & $\mu_{u_{C P L}}^{*}$ & $\overline{\text { GPL [kW] }}$ \\
\hline$[4, I V]$ & 0.7758 & 0.9850 & 550.5 & 0.9689 & 14280.550 & 0.9757 & 1.190 & 0.7758 & 0.091 & 0.9470 & 575.65 \\
\hline$[1, v]$ & 0.7758 & 0.7804 & 785.48 & 0.8614 & 19345.540 & 0.9367 & 1.340 & 0.90452 & 0.190 & 0.7854 & 974.71 \\
\hline$[1, \mathrm{VI}]$ & 0.7758 & 0.8633 & 957.36 & 0.8206 & 21435.72 & 0.9089 & 1.450 & 0.98742 & 0.0590 & 0.7944 & 950.54 \\
\hline
\end{tabular}

$V_{\text {Adap }}^{[k=I V, P r e]}=\left[\begin{array}{l}R_{D}^{[k=I V, P r e]} \\ \left.\Delta_{\mu_{\{D\}}}^{k=I V, P r e]}\right]\end{array}\right]=\left[\begin{array}{l}1.1134 \\ 0.2455\end{array}\right]$

The new trajectory starts from a state/stage whose relative judgments are not adapted (the Adaptation Ratio is greater than 0 ), and decision-making level (acceptance) that does not meet the risk threshold restriction (the Acceptance Variation of the present State is greater than 0 too). In addition to the above, there still remains the point of evaluating whether the Judgment Matrix meets a logic permitted by the regulator. This could be evaluated alternatively by comparing the Yager's Vectors corresponding to the projected and present stages (associated to $\boldsymbol{M P}_{A}^{[k=I V, P r e]}$ ) in the stage $k=I V$.

\section{Conclusions}

(1) The traditional concept of an Economically Adapted System is based on the Neoclassic economics paradigm. It is related only to the productive efficiency, which implies the expansion and operation of system with a minimum cost. It assumes as well a permanent equilibrium, which is a reason for establishing that such efficiency must take place even in the future, regardless that the planning decisions are made in the present. It ignores the uncertainties, or it renders them a stochastic nature, which they do not necessarily show to have. The proposed model implies, in such a context, $a$ change of paradigm.

(2) In this alternative paradigm, featured by the fundamental uncertainty of Keynes [14] and driven by the risk, it is rather preferred to have a set of good solutions (evolution trajectories for the system) than an 'optimal' solution that is based on an inexistent equilibrium.

(3) In this context, a new model to evaluate the Dynamic Deadaptation of a Distribution System with respect to its planning for a given period of tariff control, is presented. It is applied in the simulation (study case), considering five system attributes (as merit measures) observed by regulation of Argentina. As result, the system evolves as the most satisfactory trajectory (intrinsic risk). If the extrinsic risk is based on opportunity costs of those attributes, and the constraint (2) of model is satisfied, then such trajectory guarantees the system adaptation.

(4) In terms of system adaptation for a certain present stage $k$, $V^{\text {Yager[k,Pre] }}$ can be reconstructed. However, the equation for the hierarchic composition principle: $\boldsymbol{M P}_{A}^{[k, P r e]} \times V^{\text {Yager }[k, P r e]}=$ $\lambda_{P}^{[k, P r e]} \times V^{\text {Yager }[k, P r e]}$ does not have a unique solution in $\left(\boldsymbol{M P} \boldsymbol{P}_{A}^{[k, P r e]}, \lambda_{P}^{[k, P r e]}\right)$. The approach proposed in Eq. (12), is aimed at finding a solution that also incorporates the randomness as an adaptive element of the system. In this way, and confronting the instrumentalism that is featured by the dominant Neoclassic paradigm, this work has tried to incorporate a greater realism into the methodology.

(5) The evaluation of the static adaptation of the above point is complemented by a dynamic evaluation, by taking into account the Adaptation Variation in the present stage. It introduces the disturbance that the future system trajectory may experience as a consequence of its history.

\section{Appendix A. Definition of departures from Eq. (1) and membership function of fuzzy set for each system attribute}

In all descriptions, the Regulatory Authority is the Authority of Argentina, Province of Rio Negro. All departure variables and fuzzy sets of attributes are proposed in this work, and there not exist proposal, in the regulation, about this matter from this authority. The sub-indication $[j, k]$ refer the state $j$ in the stage $k$.

\section{A.1. Global cost}

Departure $u_{G C}$

$u_{G C[j, k]}=\frac{A C_{v j j, k]}-\operatorname{Min}\left\{A C_{v j, k]}\right\}_{k-1}}{\operatorname{Min}\left\{A C_{v j, k]}\right\}_{k-1}} \quad \mu_{u_{G C[j, k]}}=e^{-\alpha \times u_{G C[j, k]}}$

where $0<\alpha \leqslant 1$, and $A C_{v j, k]}$ is the annual cost in investment (choice $v$ of equipment) plus system's maintenance in $[j, k] . \operatorname{Min}\left\{A C_{v j, k\}}\right\}_{k-1}$ is the minimum cost of transition, between stages $(k-1, k)$ applying a classic dynamic programming approach, as a reference GC for each state.

\section{A.2. Non-supplied energy}

Departure $u_{N S E}$

$u_{N S E[j, k]}=\operatorname{Max}\left\{0 ; \frac{\left(N^{\prime} E_{[j, k]}-N S E_{[k]}^{R e f}\right)}{N S E_{[k]}^{R e f}}\right\} \quad \mu_{u_{N S E j, k]}}=e^{-\beta \times u_{N S E j, k]}}$

where $N S E^{R e f}$ is a external limit for stage $k$, of $N S E$, and $0<\beta \leqslant 1$. The limit $\left.N S E_{[k]}^{R e}\right]$ is imposed for Regulatory Authority, as function of demand in each stage $k$ 


\section{A.3. Interruption rate}

Departure $u_{F I}$

$u_{F[j, k]}=\operatorname{Max}\left\{0 ; \frac{\left(F F_{[j, k]}-F_{[k]}^{R e f}\right)}{F I_{[k]}^{R e f}}\right\} \quad \mu_{u_{F[j, k]}}=e^{-\delta \times u_{F[j, k]}}$

with : $F I_{[j, k]}=\sum_{i \in N I n t}\left(\frac{Q_{C T_{i j, k]}}^{o s}}{Q_{C T_{\text {Inst }}[j, k]}}\right)$

where $F I_{[k]}^{R e f}$ is a external limit for stage $k$, of $F I$, and $0<\delta \leqslant 1 ; Q_{C T_{i j k} k}^{o s}$ is the number of substations MV/LV out of service under interruption $i$, in the state $j$, stage $k$; and $Q_{C_{T_{\text {Inst }} t_{j k}}}$ is a number of total installed substations MV/LV in $[j, k]$; NInt is a total number of interruptions in $[j, k]$. The limit $F_{[k]}^{R e f}$ is imposed for the Regulatory Authority.

\section{A.4. Out-of-supply voltage}

Departure $u_{O S V}$

$u_{O S V[j, k]}=\operatorname{Max}\left\{0 ; \frac{\left(\operatorname{IOSV}_{[j, k]}-\operatorname{IOSV}_{[\mathrm{k}]}^{\mathrm{Ref}}\right)}{\operatorname{IOSV_{[k]}^{Ref}}}\right\} \mu_{u_{O S V j, k]}}=e^{-\gamma \times u_{O S V j, k]}}$

Membership Function of Fuzzy Set

where $0<\gamma \leqslant 1$ and $I O S V_{[k]}^{R e f}$ is an index limit for stage $k$, which construction require the following steps:

(a) Exist a set of penalties imposed for the Regulatory Authority to Energy Supplied in Non-Quality Conditions (ESNQC). This penalty, depends on two factors: (a) Density of Distribution (Urban [Ur] or Rural [Ru] areas) and (b) maximum voltage drop in the feeders $\left(\Delta u^{u}=\left|\frac{u-u_{\text {nom }}}{u_{\text {nom }}}\right|\right)$. Tables 1 and 2 show penalties corresponding, depending of limits in the factor (b), $\left(\Delta u^{u}\right)$, for each distribution area (Urban and Rural), which have an Energy Supplied $E^{\text {Total }}$ in $[j, k][U r / R u]$.

(b) With this penalties, it construct an auxiliary index, which to weight the energy supplied estimated (EfOSV $V_{[j, k \mid R u}^{R e, i}$, $E f O S V_{[j, k \mid U r}^{R e f, i}$ ) in all nodes, $i$, in each area (Urban, $n N O S V_{[j, k] U r}$ - Rural, $n N O S V_{j, k] R u}$ ) (see Tables 1 and 2), on an average

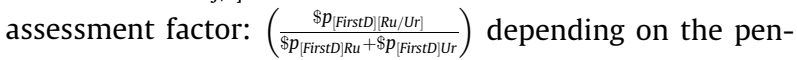
alty applied to first step (departure of $\Delta u^{u}$ ) and the total energy supplied. Then:

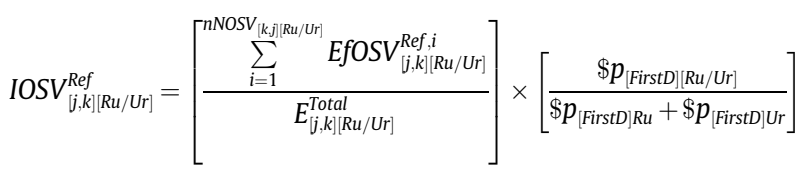

(c) Adding the minimum value obtained from both auxiliary index, in each stage $k$, it obtained a reference composite index:

$$
\operatorname{IOSV} V_{[k]}^{R e f}=\operatorname{Min}\left\{\operatorname{IOS} V_{[k, j] U r}^{R e f}\right\}_{\forall j \in k}+\operatorname{Min}\left\{\operatorname{IOSV}_{[k, j] R u}^{R e f}\right\}_{\forall j \in k}
$$

(d) Then, the index $\operatorname{IOSV}_{[j, k]}$ can be calculated to each departure of $\Delta u^{u}$, with the number of nodes corresponding, through Eq. (A.6) (for separate, Urban and Rural and applying the corresponding penalty):

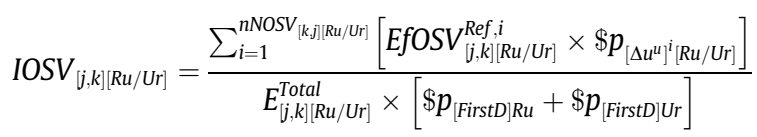

Then, adding the results, the departure Index is compound as:

$$
\operatorname{IOSV}_{[j, k]}=\operatorname{IOSV}_{[j, k]_{U r}}+\operatorname{IOSV}_{[j, k]_{R u}}
$$

The number of nodes $n N O S V_{[k, s] U r / R u}$ is estimated by means an AC power flow in the sub-system MV (13.2 [kV]) in study case. Therefore, each substation MV/LV (13.2/0.38 [kV]), become an evaluation node. In addition with this Index, are accepted voltage violations, without penalties, only in the first departure of $\Delta u^{u}$ for both areas [Ur/Ru]. This is based on that the first step of penalties, is imposed only as reference and, in practice, non-penalty is applied. The substations MV/LV under evaluation, have a typical loads curve. From this, the corresponding ESNQC is estimated for each node.

\section{A.5. Global power loss}

Departure $u_{G P L}$ Membership Function of Fuzzy Set

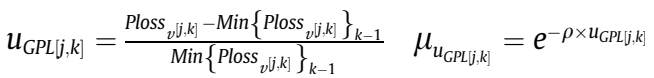

where $0<\rho \leqslant 1$, and $\operatorname{Min}\left\{\text { Ploss }_{v j, k]}\right\}_{k-1}$ are minimal power losses of transition, between stages $(k-1, k)$, obtained from a classic dynamic programming approach, as a reference Ploss, for each state.

\section{References}

[1] Bellman R, Dreyfus E. Applied dynamic programming. Princeton University Press; 1962.

[2] Bellman R, Zadeh L. Decision-making in a fuzzy environment. Manage Sci 1970;17:141-64.

[3] Saaty TL. A scaling method for priorities in hierarchical structures. J Math Psychol 1977; 15:234-81.

[4] Saaty TL. The analytic hierarchy processes. New York: McGraw-Hill; 1980.

[5] El-Fouly THM, Zeineldin HH, El-Saadany EF, Salama MMA. A new optimization model for distribution substation stiting, sizing, and timing. Int J Electr Power Energy Syst 2008;30(5):308-15.

[6] Kaur D, Sharma J. Optimal conductor sizing in radial distribution system planning. Int J Electr Power Energy Syst 2008;30(4):261-71.

[7] Miranda V, Srinivasan D, Proença LM. Evolutionary computation in power systems. Int J Electr Power Energy Syst 1998;20(2):89-98.

[8] Carvalho PMS, Ferreira LAFM, Lobo FG, Barruncho LMF. Optimal distribution expansion planning under uncertainty by evolutionary decision convergence. Int J Electr Power Energy Syst 1998;20(2):125-9.

[9] Lax PD. Linear algebra. New York: Wiley Interscience; 1997. p. 196 [chapter 16].

[10] Yager RR. Multiple objective decision making using fuzzy sets. Int J Man-Mach Stud 1977;9:53-64.

[11] Schweickardt G, Andreoni A, Miranda V. Evaluation of risk bounded distribution expansion cost with a fuzzy dynamic programming approach. In: Proceedings of the 14th PSCC'02. Sevilla, Spain; 2002.

[12] Schweickardt G, Miranda V. A fuzzy dynamic programming approach for evaluation of expansion distribution cost in uncertainty environments. J LAAR 2007;37(4):227-34.

[13] Miranda V, Proença LM. Why risk analysis outperforms probabilistic choice as the effective decision support paradigm for power systems. IEEE Trans Power Syst 1998;13(3).

[14] Lavoie M. Foundations of PostKeynesian economic analysis. Edward Elgar Publishing; 1992. 